Received: 2018.02.13 Accepted: 2018.05.08 Published: 2018.07.16

Authors' Contribution:

Study Design A

Data Collection B

Statistical Analysis $C$

Data Interpretation D

Manuscript Preparation E

Literature Search F

Funds Collection G

\section{Serotonin Syndrome in the Perioperative Setting}

Department of Anesthesiology and Perioperative Medicine, Mayo Clinic, Rochester, MN, U.S.A.
Corresponding Author: Conflict of interest:
Nathan J. Smischney, e-mail: smischney.nathan@mayo.edu None declared

\begin{abstract}
Patient: Male, 70
Final Diagnosis:

Symptoms:

Medication:

Clinical Procedure:

Specialty:

Objective:

Background:

Case Report:

Conclusions:

Serotonin syndrome

Myoclonus $\bullet$ rigidity

Photoseletive vaporization of prostate

Anasthesiology

\section{Mistake in diagnosis}

Serotonin syndrome is a life-threatening condition that can lead to neurologic complications and is associated with the use of serotonergic medications. As the use of antidepressant medications has increased, the incidence of perioperative serotonin syndrome has transitioned from a rare diagnosis to one that should be considered as a differential diagnosis for any patient displaying signs of neuroexcitation.

A 70-year-old man (ASA 2) with a history of vestibular migraines (treated with venlafaxine), gastroesophageal reflux disease, and benign prostatic hyperplasia presented to our institution for photoselective vaporization of the prostate. Upon review of prior anesthetic records, his medical chart was found to list a propofol allergy. In discussion with the patient, he stated the reaction was rigidity. The anesthesiologist and patient agreed this was not an allergy. Thus, the patient was induced with propofol and given ketamine and fentanyl boluses throughout the procedure. During emergence, the patient exhibited myoclonic jerks in the upper and lower extremities. He was given intravenous meperidine for postoperative shivering; minutes after administration, the myoclonic jerks and rigidity worsened. The anesthesia team raised concern about serotonin syndrome. Intravenous midazolam improved the patient's myoclonic jerks and rigidity.

Patients with a history of rigidity/movement disorders during the perioperative period may have experienced serotonin toxicity. It is possible, as in our case, for this history to have been labelled as an allergy to a perioperative medication. Clinicians should remain vigilant for patients at risk of developing serotonin syndrome, such as those taking outpatient medications that increase neuronal serotonin.
\end{abstract}

MeSH Keywords:

Full-text PDF:

\section{Anesthesia • Muscle Rigidity • Myoclonus • Propofol • Serotonin Syndrome}

https://www.amjcaserep.com/abstract/index/idArt/909497

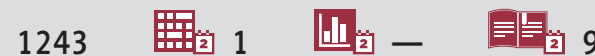




\section{Background}

Serotonin syndrome manifests as a wide spectrum of clinical presentations, which can range from self-limiting to lifethreatening. Symptoms include agitation, clonus, tremor, hypertonia, and diaphoresis. The number of cases of serotonin syndrome reported has increased over the past several years, likely due to both the increased use of serotonergic agents and increased diagnosis of the syndrome [1,2].

\section{Case Report}

A 70-year-old man (ASA 2) with a history of benign prostatic hyperplasia, vestibular migraines (treated with high-dose venlafaxine), and gastroesophageal reflux disease presented to the outpatient surgery center for a planned green-light photoselective vaporization of the prostate. Review of his medical chart prior to anesthesia revealed a listed allergy to propofol. It was determined the allergy was listed after 2 prior anesthetics when he woke up with shaking in the post-anesthesia care unit (PACU) at outside facilities. After discussion about his prior anesthetic history, the anesthesiologist and patient agreed this was not an allergy to propofol, but more likely an adverse effect of propofol, an adverse effect of another medication given during the perioperative period, or possibly not related to medication administration at all. The patient was subsequently induced with propofol $200 \mathrm{mg}$ and was given ketamine $20 \mathrm{mg}$ and fentanyl boluses for a total of $225 \mathrm{mcg}$ throughout the 2-hour procedure. He had no complications during the anesthetization or procedure.

Upon awakening after the procedure, it was noted he had myoclonic jerks in bilateral upper and lower extremities. To treat for possible postoperative shivering, meperidine $25 \mathrm{mg}$ was administered intravenously. The myoclonic jerks became worse, and hypertonia increased after receiving meperidine. The anesthesia team raised suspicion of serotonin syndrome at this time due to the patient's outpatient regimen of venlafaxine and the intraoperative administration of fentanyl. The most commonly used diagnostic tool for serotonin syndrome is the Hunter Criteria. The anesthesia team felt confident diagnosing the patient's symptoms as serotonin syndrome secondary to fulfilling the Hunter Criteria. The patient exhibited both spontaneous clonus and diaphoresis in the setting of having multiple serotonergic medications.

Subsequently, he was administered $4 \mathrm{mg}$ intravenous midazolam. The myoclonic jerks and muscle rigidity improved shortly thereafter. After the patient returned to baseline, the anesthesiologist discussed these events with the patient, and it was felt that his prior episodes of perioperative shaking and rigidity were likely similar in nature to what we witnessed.

\section{Discussion}

Serotonin syndrome remains a diagnostic conundrum. Its presentation varies widely in severity [1]. The triad of symptoms commonly associated with serotonin syndrome includes autonomic hyperactivity, neuromuscular abnormalities, and altered mental status [1,2]. Due to numerous factors, this triad is difficult to recognize both intraoperatively and postoperatively. While several medications, including selective serotonin reuptake inhibitors (SSRIs), tramadol, meperidine, and fentanyl, have a well-established association with serotonin syndrome, there are other drug classes that can potentially result in the syndrome $[3,4]$. We present a case report of postoperative serotonin syndrome in the setting of propofol allergy reported as rigidity. While the patient did have a listed propofol allergy documented at an outside hospital, there was no record of the reaction type or severity prior to the procedure. Despite the lack of intraoperative hemodynamic changes following induction with propofol, the presence of myocloniclike movements in the PACU was highly suggestive of serotonin syndrome. To the best of our knowledge, there is only 1 reported case of serotonin syndrome related to propofol administration [5]. While that case noted intraoperative clonus, we noted myoclonus in the postoperative period as the patient emerged from anesthesia.

A recent meta-analysis by Werneke et al. highlights the difficulties associated with making the diagnosis of serotonin syndrome [1]. For example, common teachings about serotonin syndrome include hyperthermia as a presenting symptom and that onset of serotonin syndrome is quicker than that of neuroleptic malignant syndrome [3]. However, most of these findings are based on very small sample sizes. Additionally, there are 3 diagnostic criteria systems (Hunter, Sternbach, and Radomski classifications), which can cause conflicting results when applied to a case $[1,6]$. Furthermore, diffuse body movements in the intraoperative and postoperative setting could easily be confused with other conditions such as malignant hyperthermia, shivering, seizures, or propofol-induced movement disorder [5]. For these reasons, clinicians must maintain an open mind about the potential for serotonin toxicity, even if diagnostic criteria are not met [1].

Given the diagnostic challenges of serotonin syndrome, there is a risk of unknowingly administering a medication that could potentially exacerbate the condition. Serotonin toxicity in our patient manifested as myoclonic jerks in bilateral upper and lower legs. This could easily be mistaken for shivering, a common finding in the postoperative period. This patient was given meperidine to treat what was hypothesized as shivering upon arrival to the PACU, which intensified the myoclonic jerks.

Over the past 20 years, an increasing number of patients in the United States have been prescribed antidepressant medications. 
Table 1. Medications that can be associated with serotonin syndrome.

\begin{tabular}{lllll}
$\begin{array}{l}\text { Selective serotonin } \\
\text { reuptake inhibitors (SSRIs) }\end{array}$ & $\begin{array}{l}\text { Serotonin-norepinephrine } \\
\text { reuptake inhibitor (SNRIs) }\end{array}$ & Opioids & Anti-emetics & Miscellaneous \\
\hline Citalopram & Paroxetine & Fentanyl & Granisetron & Trazadone \\
\hline Fluoxetine & Venlafaxine & Tramadol & Ondansetron & Mirtazapine \\
\hline Sertraline & Duloxetine & Meperidine & Metoclopramide & Buspirone \\
\hline Escitalopram & & Methadone & \\
\hline
\end{tabular}

In $1999,6.8 \%$ of adults in the U.S. were prescribed an antidepressant and by 2012 that had increased to $13 \%$ of the adult population [7]. Selective serotonin reuptake inhibitors (SSRIs) and monoamine oxidase inhibitors (MAOIs) are commonly prescribed modern antidepressants that both cause increased serotonin at the synaptic cleft. As these medications become more prevalent, perioperative physicians will see an increasing number of patients who present for surgery with elevated serotonin. It is plausible that these patients who are tolerating outpatient therapy without symptoms will receive additional medications during the perioperative period and begin to show signs of increased neurotransmission manifesting as serotonin syndrome (Table 1) [8]. It is important to remember that taking multiple serotonergic medications produces a compounding effect that can cause serotonin syndrome. Serotonin syndrome can also present later on in the perioperative course if the patient has been given large doses of benzodiazepines that mask the physical symptoms such as rigidity. In patients for whom the risk of serotonin syndrome is high, it may be beneficial to monitor them for longer periods of time in the Post-anesthesia Recovery Unit [9].

This case highlights 2 critical points: patients with a history of perioperative rigidity or movement disorders may, in fact, be experiencing serotonin syndrome. Gathering this history in a patient taking serotonergic medications should alert the clinician to consider minimizing agents that will further increase serotonin. For at-risk patients, it is important to maintain a high index of suspicion in order to diagnose serotonin syndrome in patients taking serotonergic medications. Despite the increasing

\section{References:}

1. Werneke U, Jamshidi F, Taylor DM, Ott M: Conundrums in neurology: Dagnosing serotonin syndrome - a meta-analysis of cases. BMC Neurology, 2016; 16: 97-106

2. MacKay FJ, Dunn NR, Mann RD: Antidepressants and the serotonin syndrome in general practice. Br J Gen Pract, 1999; 49: 871-74

3. Volpi-Abadie J, Kaye AM, Kaye AD: Serotonin syndrome. Ochsner J, 2013; 13: $533-40$

4. Gollapudy S, Kumar V, Dhamee MS: A case of serotonin syndrome precipitated by fentanyl and ondansetron in a patient receiving paroxetine, duloxetine, and bupropion. J Clin Anesth, 2012; 24: 251-52

5. Davis JJ, Buck NS, Swenson JD et al: Serotonin syndrome manifesting as patient movement during total intravenous anesthesia with propofol and remifentanil. J Clin Anesth, 2013; 25: 52-54 number of patients taking serotonergic medications, most notably SSRIs, many clinicians are not aware of serotonin syndrome. In $1999,85 \%$ of general practitioners were not aware of serotonin syndrome [2]. As polypharmacy increases in aging patient populations, it is important to consider serotonin syndrome in patient taking proserotonergic agents, especially in the perioperative setting, with a documented history of propofol allergy described as rigidity/movement disorder. If high-risk patients could be identified pre-operatively, they could be more safely managed by avoiding the addition of other serotonergic medications [6].

\section{Conclusions}

In summary, serotonin syndrome can be manifested as a wide variety of neurologic symptoms, agitation, and autonomic dysfunction. The diagnosis is entirely clinical, and the Hunter Criteria can be applied to assist with accurate diagnosis. Timely and supportive therapy, removal of offending agents, and cyproheptadine are mainstays of treatment. In the perioperative period, as in our case, the offending agents were likely fentanyl plus long-term venlafaxine outpatient therapy. Fortunately, fentanyl is short-acting and our patient's serotonergic toxicity was short-lived.

\section{Statement}

This work was supported by the Department of Anesthesiology and Perioperative Medicine with no direct financial support.

6. Boyer EW: Serotonin syndrome (serotonin toxicity). In: Traub SJ, Grayzel J (eds.), UpToDate. Waltham, MA, 2010

7. Kantor ED, Rehm CD, Haas JS et al: Trends in prescription drug use among adults in the United States from 1999-2012. JAMA, 2015; 314: 1818-31

8. Pedavally S, Fugate JE, Rabinstein AA: Serotonin syndrome in the Intensive Care Unit: Clinical presentation and precipitating medications. Neurocrit Care, 2014; 21: 108-13

9. Warner ME, Naranjo J, Pollard EM et al: Serotonergic medications, herbal supplements, and perioperative serotonin syndrome. Can J Anaesth, 2017; 64: $940-46$ 